\title{
Comparative Study between "Onlay" Versus "Retrorectus" Hernioplasty in Management of Uncomplicated Venteral Hernias
}

\author{
Seif El-Deen Mahmoud Alsoudany, Osama Osman Ali Khalil, Ahmed Mohamad Abdel- \\ Ghany Shebl* \\ Department of General Surgery, Faculty of Medicine, Al-Azhar University \\ *Corresponding author: Ahmed Mohamad Abdel-Ghany Shebl, Mobile: 01068490854; \\ E-Mail: sheblphone@gmail.com
}

\begin{abstract}
Background: Hernias of the anterior abdominal wall, or ventral hernias have a various types that can be categorized into either congenital or acquired, also can be categorized according to various locations into primary ventral hernias (true ventral- non incisional hernias) which include two subtypes lateral ventral hernia, and midline ventral hernias. Objective: The aim of the study was to compare between two techniques of mesh placement in uncomplicated ventral hernias, onlay (mesh on external oblique) versus sublay (mesh in the retromuscular space), regarding the operative technique and the postoperative complications. Patients and Methods: In this study, a comparative study between two methods of surgical treatment for ventral hernia was made. The study included 40 adult patients with uncomplicated ventral hernia divided randomly into two groups according to the surgical technique used for the repair, without any specific criteria used in selection for any technique as follows: Group A (Onlay mesh repair): Twenty patients were operated by placing the mesh above the anterior rectus sheath and the external oblique muscle. Group B (Sublay "Reteromuscular" mesh repair): Twenty patients were operated by placing the mesh in the retro- muscular space. Results: In this study no significant difference found between both methods as regarding: Age and gender, type of ventral hernia. Duration of the operative procedure. Amount of intraoperative blood loss. Postoperative hospital stay. Hernia recurrence. Conclusion: Sublay (Retromuscular) mesh repair is a good alternative to onlay mesh repairs, this study advocates this method of ventral hernia repair as it is applicable to all sites of ventral hernia, the mesh is mostly hidden and anchored behind the rectus sheath, the complication rate is low and there is a low recurrence rate and finally We suggest carrying out more trials on the retromuscular mesh repair technique to include a bigger number of cases and a longer period of follow up.
\end{abstract}

Keywords: Onlay, retrorectus, Laparoscopic ventral hernia repair

\section{INTRODUCTION}

Ventral hernias of the anterior abdominal wall either de novo or recurrent are relatively a common surgical problem, defined as any fascial defect of the anterolateral parietal abdominal wall fascia and muscles, through which intermittent or continuous protrusion of intra-abdominal or preperitoneal contents occurs ${ }^{(1)}$.

These hernias have a various types that can be categorized into either congenital or acquired, also can be categorized according to various locations into primary ventral hernias (true ventralnon-incisional hernias) and incisional hernias (acquired hernias); occur at the site of a pervious surgical scar. Both types have two subtypes, lateral and midline ventral hernias ${ }^{(2)}$.

The cause of a primary ventral hernia is far from completely understood, but it is undoubtedly multifactorial. Familial predisposition plays a role ${ }^{(3)}$.

Ventral hernia also can be categorized according to their characteristics into reducible, irreducible or incarcerated, strangulated and recurrent ventral hernia ${ }^{(4)}$.

They are considered as a leading cause of abdominal surgery account for $15-20 \%$ of all abdominal wall hernias ${ }^{(5)}$.

Most studies now support the theory that acute fascial separation occurs 
early in the postoperative period leading to the delayed clinical development of abdominal wall incisional hernias ${ }^{(6)}$.

Clinical data show that $52 \%$ of incisional hernias occur within 6 months postoperatively as a result of excessive tension and inadequate healing of a previous incision. Obesity, advanced age, malnutrition, ascites, pregnancy, and conditions that increase intra-abdominal pressure are factors that predispose to the development of an incisional hernia ${ }^{(7)}$.

The history of prosthetic repair in abdominal wall hernias began in 1844 by the use of silver wire coils placed on the floor of the groin to incite an inflammatory fibrosis augmenting the repair ${ }^{(8)}$.

Many prosthetic materials have been tried in hernia repair, but the two most common in current use are polypropylene mesh and expanded poly tetra flouro- etheylene (ePTFE) ${ }^{(9)}$.

Ventral hernias repair vary from primary closure only, primary closure with relaxing incisions, primary closure with onlay mesh reinforcement, onlay mesh placement only, inlay mesh placement and intraperitoneal mesh placement ${ }^{(10)}$.

Primary closure techniques usually are performed for small fascial defects less than $5 \mathrm{~cm}$ in greatest diameter. Even for small hernia defects, recurrence rates in excess of $50 \%$ have been reported (I). An onlay, usually of polypropylene mesh, is sutured to the anterior rectus sheath after fascial defect has been closed primarily. The potential advantage of this repair keeps the mesh separated from the abdominal contents by full abdominal muscle fascial wall thickness. The disadvantages of this repair include a repair under tension, large subcutaneous dissection that allows for seroma formation, and mesh infection when the surgical wound becomes infected.

The sublay (reterorectus) placement of mesh, more commonly known as Stoppa technique, became popular during 1990. The recurrence rates with this repair have been stated to be less than $10 \%^{(10)}$.

\section{AIM OF THE WORK}

The aim of the study is to compare between two techniques of mesh placement in uncomplicated ventral hernias, onlay (mesh on external oblique) versus sublay (mesh in the retromuscular space), regarding the operative technique and the postoperative complications.

\section{Patients and Methods}

Our study carried out in General Surgery Department, Menoufiya University Hospitals and Tala General Hospital from October 2012 to October 2013. It included 40 adult patients who had uncomplicated ventral hernia either De novo or recurrent.

All patients with the complaints of a lump, which appears on straining and disappears on lying down, pain, discomfort, either due to primary ventral hernia or even at the site of a scar of previous operation irrespective of gender, weight and duration of illness, were enrolled in this study according to the following inclusion and exclusion criteria:

Inclusion criteria: Having no other serious disease / illness including hemorrhagic disorders. Patients with uncomplicated ventral hernias only. Had agreed to undergo operation following either onlay mesh repair or sublay (retromuscular) mesh repair methods.

Exclusion criteria: Patients with inflamed, obstructed or strangulated ventral hernias. Very large ventral hernia defects that need special considerations before surgical interference such as components separation technique (CST), tissue expansion assisted closure and vacuum-assisted closure (VAC) therapy.

Ethical considerations were covered by standard pre-operative consent following proper instructions/guidelines from the Ministry of Health (clinical/surgical methods, biological samples/laboratory tests, etc...).

All patients were subjected to preoperative full history taking including personal history including name, age, sex, residence, occupation, marital status, 
special habits of medical importance and menstrual history for females, analysis of the main complaint either a lump, pain or incarceration under a scar of previous operation. A detailed present history was taken regarding the onset, duration and course of symptoms and past history of previous operations and its postoperative events (wound infection, ileus, distention, wound dehiscence and respiratory complications), chronic diseases (as cardiac diseases, Diabetes Mellitus...etc.), drug allergy \& intake and blood transfusion.

Our patients were subjected to preoperative clinical examination including general examination for vital signs and other systems to assess fitness for surgery and anesthesia. Local examination (abdominal examination) was done focusing on the site of the hernia, size of the defects and association of divercation and its extent, type of healing (primary or secondary intention, keloidal tendency), irreducible or not and if there is any other signs of hernia complications(infection, obstruction, strangulation,...etc.). In addition, the abdomen was examined for any organomegaly or other intra-abdominal co pathology to deal with it during hernia repair.

\section{Laboratory}

(Routine)

investigations were done for all patients including complete blood count (CBC), ALT, AST, Urea, Creatinine, blood sugar, P.T and serum albumin also radiological investigations such as abdominal ultrasonography to exclude any intraabdominal co-pathology and Chest plain $\mathrm{x}$-ray study in cases of previous history of smoking, bronchial asthma, or clinical signs of chest troubles.

The patients were divided randomly by the use of closed envelope method into two groups according to the surgical technique used for the treatment of the uncomplicated ventral hernia as follows:

Group A (Onlay mesh repair): Twenty patients were operated by placing the mesh above the anterior rectus sheath and the external oblique muscle. Group B (Sublay "Reteromuscular" mesh repair): Twenty patients were operated by placing the mesh in the retro- muscular space.

Patients were admitted the day before surgery except for diabetic patients who were admitted 2 days before operation for control of blood sugar. The night before surgery, preparation of the site of the operation then hair shaving just before operation at the morning. A single dose of broad spectrum antibiotic given with induction of anesthesia. General anesthesia with endotracheal intubation or spinal anesthesia was used. All patients were placed in the supine position. Operative field was sterilized by povidone-iodine and toweled up in normal manner.

\section{RESULTS}

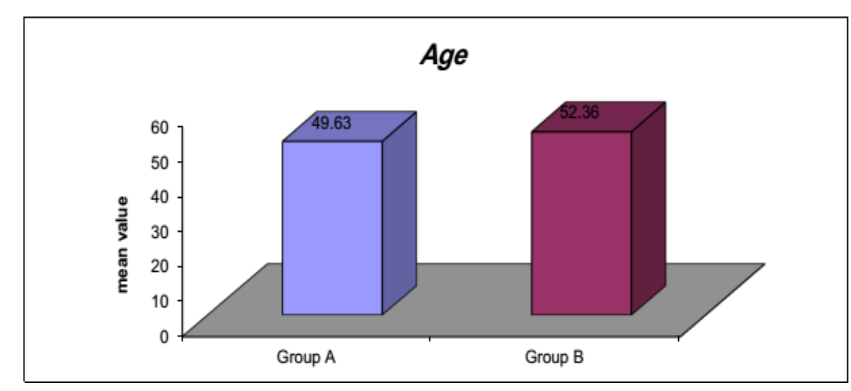

Fig. (1): Distribution of age in studied patients.

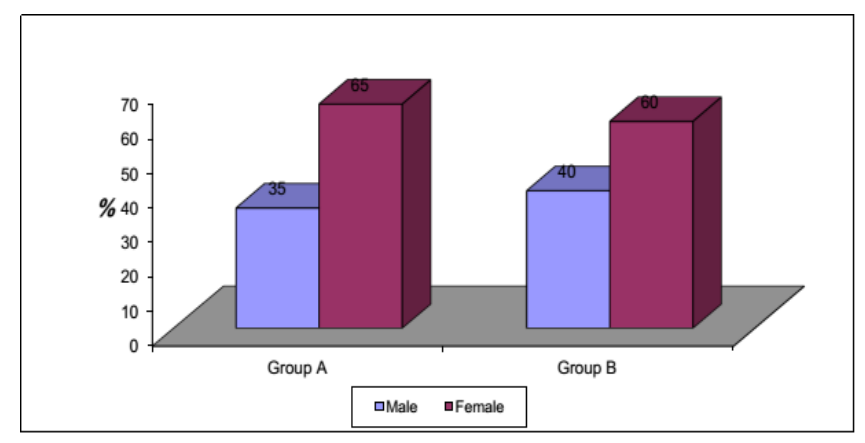

Fig. (2): Distribution of sex in studied patients. 


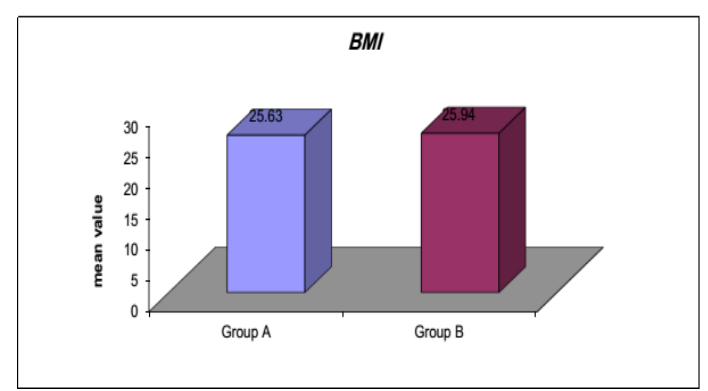

Fig. (3): Body mass index in studied patients.

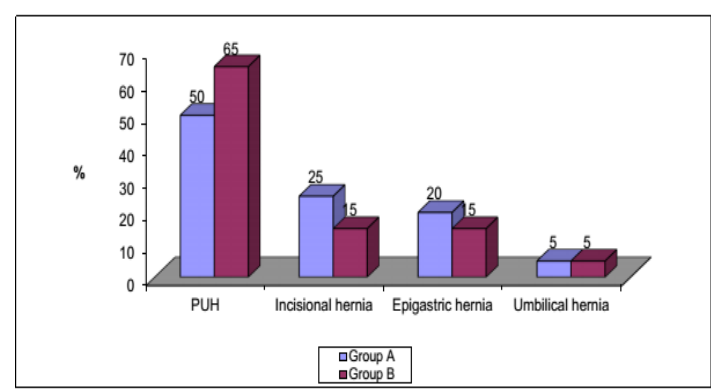

Fig. (4): Type of the hernia in studied patients.

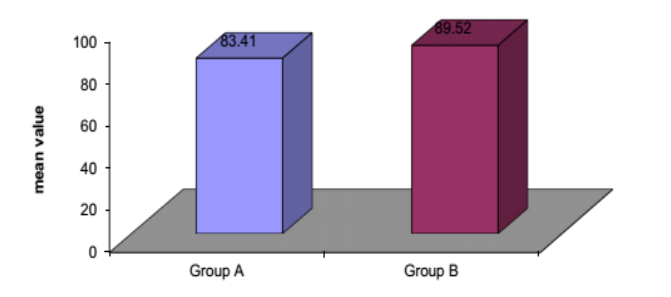

Fig. (5): Distribution of patients regarding duration of surgery in minutes.

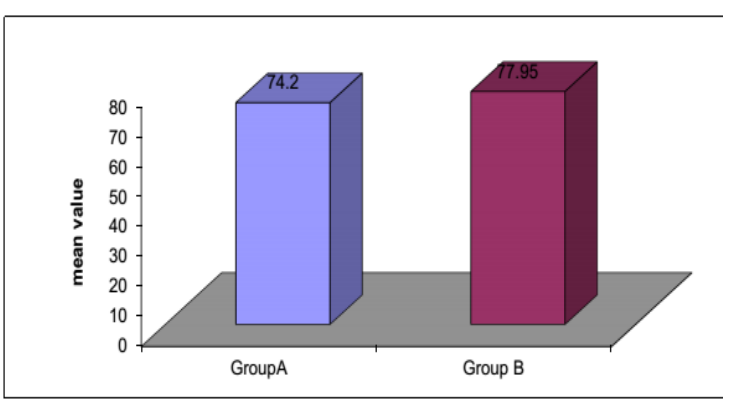

Fig. (6): Distribution of patients regarding amount of blood loss in cc.

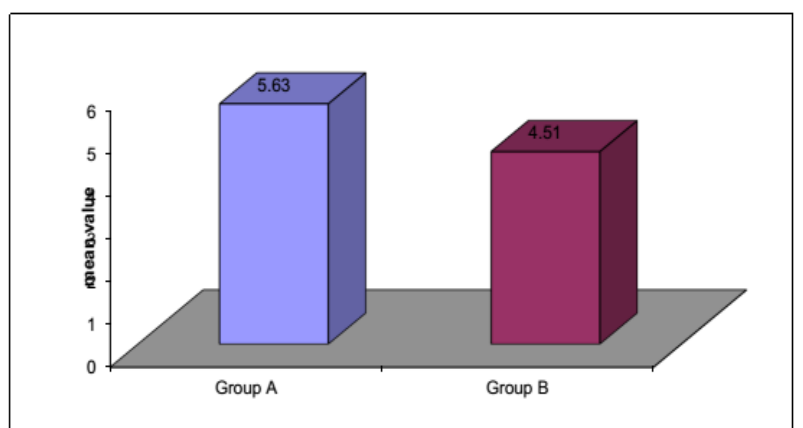

Fig. (7): Distribution of time to remove drain in both groups.

\section{DISCUSSION}

Ventral hernia either De novo or recurrent is a common surgical problem and refers to fascial defect of the anterolateral parietal abdominal wall fascia and muscles, through which intermittent or continuous protrusion of intra-abdominal or preperitoneal contents occurs $^{(1)}$.

Based on national operative statistics, ventral hernia commonly encountered in surgical practice accounts for $15-20 \%$ of all abdominal wall hernias. These defects can be categorized into either congenital or acquired, also can be categorized according to various locations into primary ventral hernias (true ventralnon-incisional hernias) which include two subtypes lateral ventral hernia, otherwise called spigelian hernia and midline ventral hernias ${ }^{(5)}$.

Incisional hernias (acquired hernias) is another type according to the location, occur at the site of a pervious surgical incision after an abdominal operation. It represents the most common wound complication after abdominal surgery, with a reported incidence rate of $2 \%$ to $11 \%$ and a recurrent herniation rate of $20 \%$ to $46 \%{ }^{(11)}$.

Numerous studies were done to understand the hernial mechanism and the methods of repair. All studies stressed on managing hernial defects as a part of generalized abdominal wall problem ${ }^{(12)}$.

Careful evaluation of the patient, who presents with an abdominal defect, reveals predisposing factors for herniation 
including inadequate local fascial and muscular layers due to prior tissue loss, muscle denervation or vascular insufficiency due to prior irradiation, wound infection, obesity, chronic pulmonary disease, malnutrition, sepsis, anemia, corticosteroid dependency and/ or current malignant process. All patients demonstrated one or more risk factors that predispose to problems with abdominal closure $^{(13)}$.

Repair of ventral hernia is an ongoing challenging in surgery. A wide spectrum of surgical techniques, have been developed and recommended, ranging from the direct suture techniques to the use of various types of prosthetic mesh. They are all aiming to close the defect and to strengthen the musculofascial tissues to avoid recurrence ${ }^{(14)}$.

Many surgical techniques were advocated however, there is still doubt about the ideal and best method that provide the least incidence of recurrence and apply patient satisfaction. Local repair without the use of mesh resulted in many recurrences. The abdominal wall becomes more destroyed and weakened making future attempts of repair more difficult ${ }^{(15)}$.

The use of surgical mesh has a dramatic reduction in the incidence of recurrence reaching to $1.8 \%$ in some studies. The use of sheets of nonabsorbable mesh prosthesis placed across the defect and fixed to the abdominal wall has rendered most of the older types of operations obsolete $^{(16)}$.

Various prosthetic materials are available; the most popular is the monofilament polypropylene mesh. A series of laboratory and clinical investigations reporting that polypropylene stimulates a strong fibroblastic response and has a dramatic resistance to infection (17).

In this study there was no statistical significant in regarding to age, gender and type of ventral hernia between two studied groups.

Regarding to the duration of surgery in patients treated with onlay mesh repair (Group A) ranged from 75-90 minutes $(83.41+10.24)$ which is longer than that reported by Godara et al. ${ }^{(11)}$ of which the duration of surgery ranged from 30 - 90 minutes $(49.35 \pm 8.29)$.

We recorded the duration of surgery in patients treated with retromuscular mesh repair (Group B) that ranged from 80-100 minutes $(89.52+7.25)$ which is slightly longer than reported by Godara et al., 2006 of which the duration of surgery ranged from $36-96(63.15 \pm 15.0)$. This was attributable to the relatively difficult dissection at the reteromuscular plane.

In our study the amount of blood loss among group A ranged from 50-100cc $(74.2+12.36)$, which is slightly less than among group $B$ which ranged from 50 $120 \mathrm{cc}(77.95+15.6)$, probably related to the extent of dissection.

Drain was removed in patients treated with onlay mesh repair (Group A) after a period of time ranged from 4-9 days $(5.63 \pm 2.14)$ which is slightly higher than that reported by Godara et al. ${ }^{(11)}$ of which the period of drainage ranged from 2-7 days and slightly higher than that reported by Bauer et al. ${ }^{(18)}$ of which the mean duration of drainage was 5 days.

While in patients treated with retromuscular mesh repair (Group B), drain was removed after a period of time ranged from 2-6 days $(4.51 \pm 0.95)$ which is comparable to that reported by Kohler et al., 2005 of which the period of drainage ranged from 2- 5 days $(2 \pm 0.8)$, but lower than to that reported by Godara et al. ${ }^{(11)}$ of which the period of drainage ranged from 2-8 days and lower than that reported by Hameed et al. ${ }^{(19)}$ of which the period of drainage ranged from 3-8 days with the average period being 4- 6 days.

Seroma is one of the most common complications following open technique and is particularly likely to occur when large skin flaps are developed during the surgical procedure. Although small seromas frequently resolve within 6 to 8 weeks without sequelae, a large symptomatic or persistent seroma occasionally requires 
multiple aspirations with subsequent increased risk for secondary infection ${ }^{(20)}$.

In this study regarding Seroma formation after drain removal in patients treated with onlay mesh repair (Group A), seroma occurred in 3 patients (15\%) which is similar to that reported by Godara et al. (11) of which $15 \%$ of cases developed wound seroma, but higher than that reported by Kohler et al. ${ }^{(20)}$ of which $12.5 \%$ of cases developed wound seroma and higher than the study done by Bauer et al. ${ }^{(21)}$ that showed a $5 \%$ incidence of seroma.

Seroma formation after drain removal in patients treated with retromuscular mesh repair (Group B), occur in only one of the cases of this group (5\%) which is higher than that reported by Kohler et al. ${ }^{(20)}$ and higher than that reported by Hameed et al. ${ }^{(19)}$ of which seroma occurred only in one patient (2\%), but lower than that reported by Bauer et al. ${ }^{(21)}$ in which seroma occurred in about $12.3 \%$ of cases and than that reported by Godara et al., 2006 in which seroma occurred in about $22.5 \%$ of cases. Which may be attributable to the relatively small number of cases in our study. Obese patients, wide areas of dissection, and the presence of devitalized tissues are conditions favoring infection which is a real threat to the successful repair, when suppuration occurs in the wound, drainage and proper antibiotics are essential ${ }^{(22)}$.

Postoperative wound infection in patients treated with onlay mesh repair (Group A), 3 patients (15\%) developed wound infection (One of them was a major wound infection that necessitated mesh removal and the other ones showed good response to conservative treatment), which is similar to that reported by Godara et al. ${ }^{(11)}$ of which $15 \%$ of cases developed wound infection and lower than that reported by Bauer et al ${ }^{(18)}$ who reported that wound infection occurred in $23 \%$, but higher to that reported by Kohler et al. (23) of which wound infection occurred in $9.3 \%$ of cases.

Our study shows that postoperative wound infection in patients treated with retromuscular mesh repair (Group B), one patient developed minor wound infection (5\%) which is lower than that reported by Yaghoobi et al. ${ }^{(24)}$ who reported that infection rate occurred in $11.6 \%$ of cases and that reported by Godara et al. ${ }^{(11)}$ of which $22.5 \%$ of cases developed wound infection but higher than that reported by Kohler et al. ${ }^{(20)}$ of which wound infection occurred in $2.5 \%$ of cases.

The length of hospital stay following treatment of ventral hernia by onlay mesh repair (Group A) was 3-9 days $(4.63 \pm 0.35)$ which is comparable to that reported by Godara et al. (11) with mean duration of hospital stay of (4.6 \pm 1.30 days), but longer than that reported by Bauer et al. ${ }^{(21)}$ who reported a mean hospital stay of 2 days, while following treatment of ventral hernia by retromuscular mesh repair (Group B) was 1-4 days $(2.62 \pm 0.74)$ which is lower than that reported by Godara et al. ${ }^{\text {(II) }}$ with mean duration of hospital stay of $(6.8 \pm$ 1.50 days) and that reported by Voeller and Mangiante ${ }^{(25)}$ of which the main hospital stay about 5.8 day. Hernia recurrence is distressing to the patient and embarrassing to surgeon. Nowadays tension free repair using prosthetic mesh has decreased the recurrence to negligible ${ }^{\text {(26) }}$.

Hernia recurrence was observed in patients treated with onlay mesh repair (Group A), only one patient (5\%) developed hernia recurrence (occurred after about 11 months post operation) which is lower than that reported by than that reported by Bauer et al. ${ }^{(18)}$ who reported that recurrence rate is about $10 \%$, this may be attributable to the shorter period of follow up in our study, but higher than that reported by Kohler et al. (20) who reported that recurrence rate is about $3.1 \%$ and than that reported by Godara et al. ${ }^{(I I)}$ with no recurrence after a $100 \%$ follow-up of minimum 24 months.

In this study regarding hernia recurrence in patients treated with retromuscular mesh repair (Group B), there is there is only one case of recurrence occurred among the cases of this group (5\%) (during the period of follow up that ranged 6-12months) which is higher than 
that reported by Godara et al. ${ }^{(11)}$; Bauer et al. ${ }^{(18)}$; Hameed et al. ${ }^{(19)}$; Kohler et al. ${ }^{(24)}$, which there is no recurrence occurred among the cases of this group.Previous studies have shown that $70-75 \%$ of recurrences develop within 2 years and $80-90 \%$ develops within 3 years ${ }^{(17)}$. Our follow- up, therefore, is probably not long enough and should be extended for at least another year.

These results were comparable to the results of Kohler et al. ${ }^{(20)}$, but differ from that by Bauer et al. ${ }^{(21)}$ in which no significant difference between two groups in duration of drainage and there is no significant difference between two groups regarding seroma formation after removal of the drains. Also differ from those results of Godara et al. ${ }^{(11)}$ in which there is significant difference between two groups in duration of the operative procedure and mean length of postoperative hospital stay.

\section{CONCLUSION}

Sublay (Retromuscular) mesh repair is a good alternative to onlay mesh repairs, this study advocates this method of ventral hernia repair as it is applicable to all sites of ventral hernia, the mesh is mostly hidden and anchored behind the rectus sheath, the complication rate is low and there is a low recurrence rate and finally We suggest carrying out more trials on the retromuscular mesh repair technique to include a bigger number of cases and a longer period of follow up.

\section{REFERENCES}

1. Ahmed M, Niaz A, Hussain A, Saeeduddin A (2003):

Polypropylene Mesh Repair of incisional Hernia. JCPS., 13 (8):440442.

2. De Vries Reilingh TS, Bodegom M.E, Van Goor H, et al. (2007): Autologous tissue repair of large abdominal wall defects. British Journal of Surgery, 94(7): 791-803.
3. Abramson JH, Gofin J, Hopp C et al. (2010): The epidemiology of inguinal hernia. A survey in western Jerusalem. J Epidemiol Community Health, 32:59-62.

4. Muysoms FE, Miserez M, Berrevoet F et al. (2009): Classification of primary and incisional abdominal wall hernias. Hernia, 13(4):407- 14.

5. Stumpf $M$, Conze $J$, Klinge $U$, Rosch R, Schumpelick V (2003): Open mesh repair. European Surgery, 35 (1) 21-24.

6. Dubay DA, Wang $X$, Kuhn MA, Robson MC, Franz MG (2004): The Prevention of Incisional Hernia Formation Using a Delayed-Release Polymer of Basic Fibroblast Growth Factor. Ann. Surg., 240(1): 179-186.

7. Malangoni MA and Rosen MJ (2017): Ventral hernia. Sabiston Textbook of Surgery, 18th ed. Section X, chapter 44. pennstatehershey.adam.com/content.a spx? productId $=117 \&$ pid $=1 \&$ gid $=007$ 661

8. Phelps AM (2013): A new operation for hernia, New york med. J., 60:291.

9. Murphy JL, Freeman JB, Dionne PG (2006): Comparison between Marlex and Gor-tex to repair abdominal wall defects in rats. Can. J. Surg., 32: 244-247

10. Millikan KW (2003): Incisional hernia repair. Surg Clin N Am., 83: 1223 - 1234 .

11. Godara R, Garg P, Raj H, Singla SL (2006): Comparative Evaluation Of "Sublay" Versus "Onlay" Meshplasty In Ventral Hernias. The Internet Journal of Surgery,DOI: 10.5580/ad9

12. Jenkins TPN (2003): Incisional hernia repair,a mechanical approach. Br.J.Surg., 67:335-36 
13. Wicks A, Voyvodic C, Scroop R (2000): Incisional hernia \&small bowel obstruction following laparoscopic surgery: computed tomography diagnosis Australas. Radiol., 44(3):331-332.

14. Cassar $K$ and Munro A (2002): Surgical treatment of incisional hernia. Br. J. Surg., 89:534-545.

15. Ponka JL (2003): Hernias of the abdominal wall, Incisional hernia. W.B. Saunders Co.; Philadelphia, Toronto.

16. McLanahan D, King LT, Weems C, Novotney M, Gibson K (2005): Preperitoneal prosthetic repair for midline abdominal hernias Am. J. Surg., 173(5):445-9.

17. Ahluwalia HS, Pim Burger J, Quinn TH (2004): Anatomy of the anterior abdominal wall. Operative Tech. in General Surgery, 6 (3): 147155.

18. Bauer JJ, Harris MT, Kreel I, Gelerent IM (2010): Twelve years experience with polypropylene in repair of abdominal wall defect. Mt. Sinai. J. Med., 66(1): 20-5.

19. Hameed F, Ahmed B, Ahmed A, Dab RH (2009): Incisional Hernia Repair by Preperitoneal (Sublay) Mesh Implantation A.P.M.C ., 3:2731.

20. Haytham MA, Kwan Hur, Hirter A, Kim LT, Thomas A, Berger DH, Reda D, Kamal MF (2009): Seroma in ventral incisional herniorrhaphy: incidence, predictors and outcome. The American Journal of Surgery, 198: 639-644.
21. Melman L et al. (2011): Histologic and biomechanical evaluation of crosslinked and non-crosslinked biologic meshes in a porcine model of ventral incisional hernia repair. $\mathbf{J}$ Am Coll Surg., 212(5):880-888.

22. Robert MZ (2002): Complications of general surgery Surg. Clin. N. Am.,71(6): 1359-1361.

23. Kohler L, Sauerland S, Meyer A, Saad S, Schüller BK, Knaebel HP, Seiler CM (2005): Mesh implantation in onlay or sublay technique for closure of median ventral hernias: first results of a randomized clinical trial. Poster presented at the Congress of the German Surgical Association. https://convention.visitberlin.de/.../co ngresscalendar/135rd-congress-ofgerman-societ

24. Yaghoobi NA, Farshi JS, Amoli HA, Salimi J, Mamarabadi M (2006): Outcomes of the RivesStoppa technique in incisional hernia repair: ten years of experience. Hernia, 11(1):25-9.

25. Voeller GR and Mangiante EC (2000): Laparoscopic repair of ventral - incisional hernias. Edited by Nyhus LM and Condon RE. 5th Ed. JB Lippincott Co. Philadelphia, Pennsylvania.

26. Bucknall TE, Cox PJ, Ellis H (2011): Burst abdomen and incisional hernia, a prospective study of 129 major laparotomies. Br. Med. J., 284:931-933. 